\title{
Intra- and intermale variability of mature sperm traits analysed in two brackish water populations of the pipefish Syngnathus abaster (Syngnathidae)
}

\author{
Francesca Piras, ${ }^{1}$ Francesca Biagi, ${ }^{2}$ Antonello Floris, ${ }^{2}$ Vittorio Farina, ${ }^{2}$ Marco Zedda, ${ }^{2}$ Piero Franzoi ${ }^{3}$ \\ and Marcella Carcupino ${ }^{1}$
}

\author{
${ }^{1}$ Dipartimento di Scienze della Natura e del \\ Territorio, Università di Sassari, Sassari, \\ Italy; ${ }^{2}$ Dipartimento di Medicina Veterina- \\ ria, Università di Sassari, Sassari, Italy; \\ ${ }^{3}$ Dipartimento di Scienze Ambientali, \\ Informatica e Statistica, Università Ca' \\ Foscari di Venezia, Venezia, Italy
}

Keywords:

morphometric, parasperm, sperm competition

Accepted for publication:

09 December 2014

\begin{abstract}
Piras, F., Biagi, F., Floris, A., Farina, V., Zedda, M., Franzoi, P., Carcupino, M. 2016. Intra- and intermale variability of mature sperm traits analysed in two brackish water populations of the pipefish Syngnathus abaster (Syngnathidae). - Acta Zool ogica
\end{abstract} (Stockholm) 97: 177-186.

Sperm cells are highly diversified in animals, and considerable research effort has focused on variation in sperm morphology among species. Surprisingly, little is known about intraspecific variation in sperm morphology. We analysed withinand between-male variation in mature sperm traits in two brackish water populations of the pipefish Syngnathus abaster. Four morphometric parameters, such as the width and length of the head (including nucleus, and midpiece), length of flagellum and total sperm length were taken into account. The differences in all morphometric parameters analysed between populations were not statistically significant. Moreover, the multidimensional scaling analysis shows that (i) the two populations seem to be indistinguishable based on their spermatozoa and (ii) there is not polymorphism, being sperm not distinguishable into discrete classes both within a single male and between males of each populations. The latter datum does not seem to support the presence of polymorphic sperm in syngnathids. Both populations, however, exhibit a high variation in all sperm traits, both among individual sperm within an ejaculate and among males within each population. The relationship between sperm traits variability and the low selection pressure determined by the absence of postcopulatory sexual selection (i.e. absence of sperm competition) is discussed.

Marcella Carcupino, Dipartimento di Scienze della Natura e del Territorio, Via Muroni 25, 07100 Sassari, Italy. E-mail: carcupin@uniss.it

\section{Introduction}

Commonly, male gametes in one species are unified in a single morph type, but the production of multiple morphs within a species occurs, to some extent, in both animals and plants (Till-Bottraud et al. 2005). The phenomenon of the production of multiple discrete morphological classes in male gametes within a single species is termed sperm/pollen polymorphism (heteromorphism).

In the animal kingdom, sperm polymorphism can be distinguished in the following categories: heteromorphic sperm sensu stricto, which are regularly produced through a constant developmental process during spermatogenesis, and aberrant sperm, which are irregularly formed by some errors at certain stages during spermatogenesis (Tachi 1990; Swallow and Wilkinson 2002). The former are called 'parasperm', in opposition to 'eusperm' (functional sperm) (Healy and Jamieson 1981; Jamieson 1987), and the seconds are called 'deformed sperm'. Both parasperm and deformed sperm are generally non-fertile sperm. According to Hayakawa (2007), deformed sperm must be clearly distinguished from parasperm and the main differences between them can be seen in the more regular process of development and in a lesser variability of morphological traits of the parasperm respect to deformed sperm.

The occurrence of parasperm has been widely confirmed in invertebrates: Annelida (Oligochaeta), Rotifera, Pogonophora, Mollusca and Arthropoda (Jamieson 1987; Swallow and Wilkinson 2002; Hayakawa 2007), whereas, there are few 
reports concerning parasperm in vertebrates, being, most of the polymorphic spermatozoa reported, apparently abnormal spermatozoa (Van der Horst et al. 2011).

Among teleosts, parasperm are reported only in few species, including some species of Syngnathidae family, in which the semen contained, together with functional sperm (eusperm), parasperm. Teleost parasperm varies in morphology among species. In cardinal fish (Apogonidae) and in a marine cottoid, (Blepsias cirrhosis), parasperm possessed two flagella, (Mattei and Mattei 1984; Lahnsteiner 2003 and Fishelson et al. 2006; Hayakawa 2007), whereas in another non-copulatory marine cottoid (Hemilepidotus gilbert) and in a marine sculpin (Cottus kazika), parasperm are unflagellate cells (Hayakawa 2007; Daisuke et al. 2010).

Syngnathidae is a small fish family characterized by extremely specialized paternal care; males take care of the embryos carrying eggs on the ventral surface of the body (external brooders), or inside a specialized brooding cutaneous organ (internal brooders), commonly referred as the brood pouch, (Herald 1959; Carcupino et al. 1997, 2002; Watanabe et al. 1999; Monteiro et al. 2005).

The functional spermatozoa of all syngnathids examined up to now (Carcupino et al. 1999; Watanabe et al. 2000; AhKing et al. 2006; Van Look et al. 2007; Biagi et al. 2008, 2014; Dzyuba et al. 2008) may be considered belonging to the 'introsperm type' (according to the classification of Jamieson (1991). This sperm type is a mono-flagellate cell with an elongated nucleus, and it is typical of internal fertilizing fishes (Jamieson 1991). In the testis lumen of Syngnathus schlegeli and Hippocampus kuda (Watanabe et al. 2000; Van Look et al. 2007), eusperm are seen to be free together with another kind of sperm, similar to those categorized as 'aquasperm' type. The acquasperm are characterized by a spherical head and are typical of external fertilizing fish (Jamieson 1991). Moreover, three different morphotypes, differing in flagellum length, head length and head wide, have been reported in a freshwater population of Syngnathus abaster (Dzyuba et al. 2008).

It should be pointed out that clear criteria to identify parasperm must be used in studies of parasperm in fish. Parasperm of teleosts need to be recognized by comparing cells in the cyst and within the semen, because irregularly shaped cells in the seminal fluid could be either parasperm or normal spermatids (Hayakawa 2007). Some teleosts, including syngnathids, at least those belonging to the Syngnathus genus (Carcupino et al. 1999; Biagi et al. 2015) and Phyllopteryx taeniolatus (Forsgren and Young 2009), show a spermatogenetic process of the semicystic type. This type of spermatogenesis results in an asynchronous maturation of spermatids, with the simultaneous presence of germ cells at different stages, inside the testis lumen (Mattei 1993; Hayakawa 2007; Biagi et al. 2015).

Because the semicystic spermatogenesis, at least in Syngnathus schlegeli, the acquasperm may not be parasperm but, more likely, developing spermatids, as it has been also admitted by Watanabe et al. (2000). However, Dzyuba et al.
(2008) reject this hypotheses for the shorter spermatozoa of Syngnathus abaster. These authors asserted that '. . . the sperm head dimensions were not so high variable as might have been expected if the short spermatozoa were actually developing spermatids'.

The aim of this study was to shed light on this topic examining the variation in sperm morphometric within male, within a population (between males) and between two brackish water populations of Syngnathus abaster.

\section{Materials and Methods}

\section{Samples}

Adult males of Syngnathus abaster were collected in two Mediterranean lagoons (Cabras pound, in western Sardinia and Venice lagoon, Italy). All samples $(N=12$ for Sardinian population, and $N=10$ for Venice population) were captured during the reproductive period (May-September, 2013).

\section{Sperm morphology}

Sperm morphology was analysed using both light (LM) and scanning electron microscopy (SEM).

For LM analysis, aliquots $(20 \mu \mathrm{L})$ of unfixed seminal fluid, obtained from testes of one male for each population, were placed on slides and immediately observed with a Zeiss Axiophot light microscope (ZEISS, Oberkochen, Germany) using the Nomarski interference contrast.

For SEM analysis, aliquots $(20 \mu \mathrm{L})$ of formalin-fixed seminal fluid, obtained from testes of one male of the Sardinian population, were applied onto a coverslip precoated with poly-lysine. Next, germinal cells adhering to the coverslips were dehydrated using a graded ethanol series $(5,10,15,20$, $30,40,50,60,70,80,90,95$ and $100 \%$ ethanol) and critical point dried in a Critical Point Dryer (Bal-Tec CPD 030, Bal-Tec AG, Balzers, Liechtenstein), gold-palladium coated and observed with a scanning electron microscope (JEOL JSM 5200, JEOL, Tokyo, Japan).

\section{Sperm morphometric}

Aliquots $(20 \mu \mathrm{L})$ of seminal fluid, fixed in $5 \%$ glutaraldehyde, were placed on slides precoated with poly-lysine, stained with toluidine blue to $0.1 \%$ in aqueous solution and allowed to dry to air before being analysed to obtain digital images and related measures, using an optical microscope. Digital images were acquired with a digital camera Nikon DS-fil connected with the control unit DS-L2 and mounted on an optical microscope Nikon Eclipse 80i (Nikon, Shinjuku, Japan). The measurements were made using the program Tpsdig2.

Four morphometric parameters, such as the width and length of the head (including the nucleus and the midpiece), the length of the flagellum and the total sperm length, were taken into account. The last one was calculated as the sum 
of head and flagellum length. Twenty intact sperm were analysed for each male $(N=10$ for Sardinian population and $N=9$ for Venice population). Sperms damaged, or difficult to measure, were discarded. As a standardized measure of variation, the coefficient of variation $(\mathrm{CV}=(\mathrm{SD} /$ mean $) \times 100)$ was used, symbolizing as $\mathrm{CVbm}$ the between-male $\mathrm{CV}$ in mean sperm traits and CVwm the mean within-male $\mathrm{CV}$ in sperm traits. This was carried out for all males in all populations (Table 1). Given that the CV of small samples tends to be underestimated, the formula of CVbm was adjusted as suggested by Sokal and Rohlf (1995) and by Laskemoen et al. (2007) in the following way: CVadj $=\mathrm{CV} \times(1+(1 / 4 \mathrm{~N}))$.

\section{Statistical analyses}

The averages of all parameters in the two populations were compared using the $t$-Student. Moreover, a metric multidimensional scaling (MDS) to examine the differences in sperm population was carried out using the PRIMER statistical package version 6.0. Differences within a single male were estimated analysing 30 sperm, obtained by a single male randomly chosen among Sardinian males. Differences within each S. abaster populations were estimated analysing 200 sperm for the Sardinian population and 180 for the Venice population. Differences between populations were estimated analysing 200 sperm obtained pooling 100 sperm, randomly selected, for each population.

\section{Results}

The morphological analysis showed that mature and normal sperm of both population analysed were very similar. All analysed mature sperm were mono-flagellate cells characterized by a cylindrical head, entirely occupied by the nucleus, followed by a short midpiece, particularly visible in the SEM images (Fig. 1A-C). Due to semicystic spermatogenesis, fulldeveloped sperm and developing spermatid are both free in the testis lumen (Fig. 1D). The latter is uni- and more
Fig. 1-Full-developed sperm, developing spermatids and damaged sperm of Syngnathus abaster. - A, B. Scanning electron micrographs of mature sperm obtained by a male of Sardinian population. - C. Toluidine Blue stained mature sperm obtained by fixed seminal fluid of one male of Venice population. D, E. Nomarski interference contrast micrographs of developing spermatid with two (D) and four (E) nuclei and flagella, obtained by unfixed seminal fluid of one male of Venice population. - F. Damaged toluidine Blue stained mature sperm obtained by fixed seminal fluid of one male of Sardinian population. Flagellum (F), nucleus $(\mathrm{N})$, sperm midpiece (MP). Polynucleated cells (PC), spermatozoon (Sp). Scale bar: A $=3 \mu \mathrm{m}$; B $=1.8 \mu \mathrm{m}$, $\mathrm{C}=10 \mu \mathrm{m}, \mathrm{D}=4 \mu \mathrm{m}, \mathrm{E}=8.3 \mu \mathrm{m}$, $\mathrm{F}=4.5 \mu \mathrm{m}$
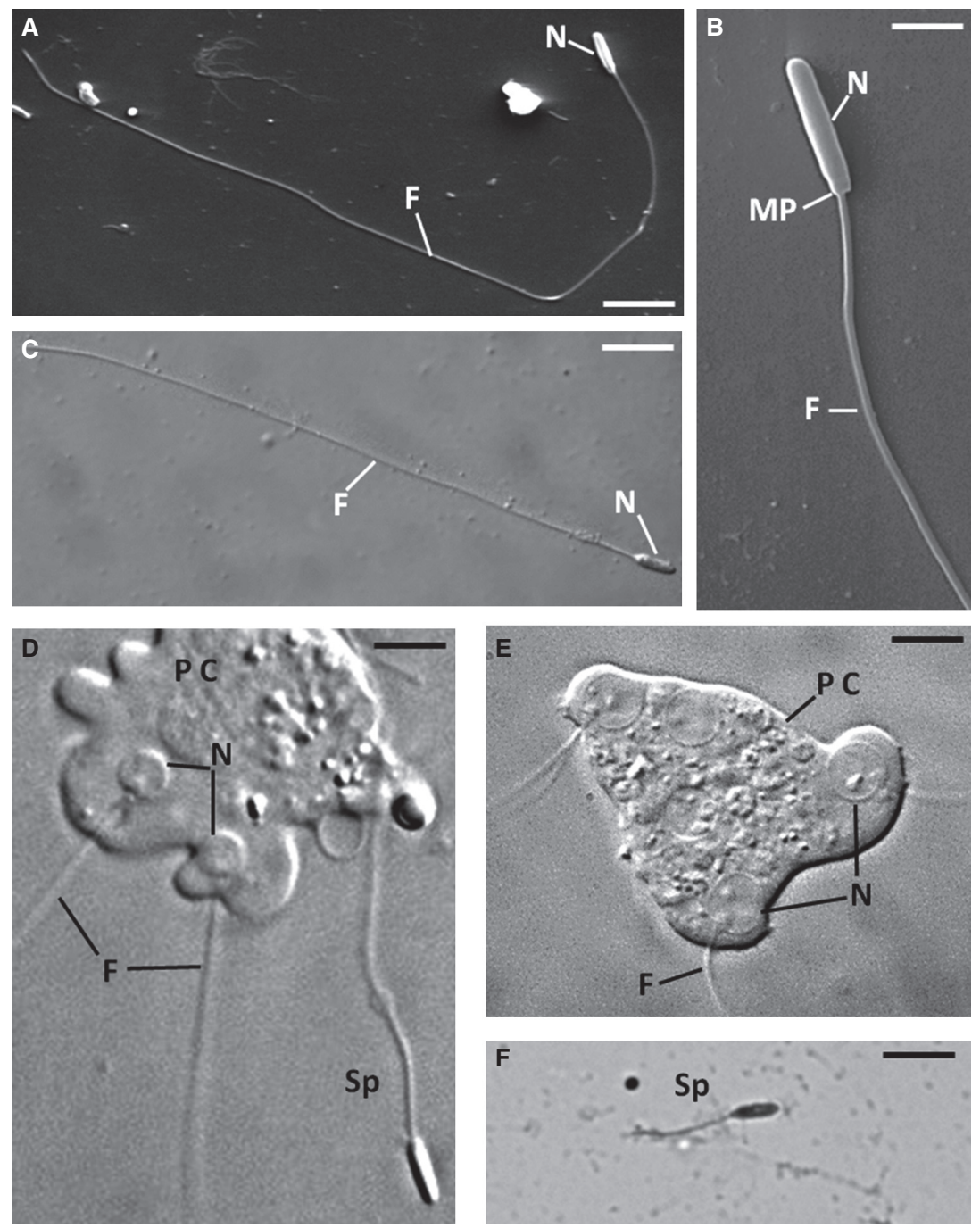


\begin{tabular}{lccl}
\hline Sperm traits & S. abaster Sardinian $N=10$ & S. abaster Venice $N=9$ & $t$-Student \\
\hline Head width (HW) & $0.75 \pm 0.12 \mu \mathrm{m}$ & $0.70 \pm 0.12 \mu \mathrm{m}$ & $t=1.66 ; P=0.113$ \\
Head length (HL) & $3.27 \pm 0.26 \mu \mathrm{m}$ & $3.25 \pm 0.14 \mu \mathrm{m}$ & $t=4.52 ; P=0.38$ \\
Flagellum length (FL) & $55.13 \pm 6.35 \mu \mathrm{m}$ & $54.15 \pm 4.28 \mu \mathrm{m}$ & $t=2.64 ; P=0.676$ \\
Total sperm length (TL) & $58.4 \pm 6.44 \mu \mathrm{m}$ & $57.55 \pm 7.49 \mu \mathrm{m}$ & $t=1.26 ; P=0.22$ \\
HW CV & $13 \mathrm{wm}$ & $15.22 \pm 3.37 \mu \mathrm{m}$ & $t=0.639 ; P=0.53$ \\
HW CV & 16.4 & 17.61 & \\
HL CV & $7.56 \mu \mathrm{m}$ & $7.73 \pm 1.73 \mu \mathrm{m}$ & $t=0.218 ; P=0.83$ \\
HL CV & $7.07 \pm 1.66 \mu \mathrm{m}$ & $7.17 \pm 2.38 \mu \mathrm{m}$ & $t=0.377 ; P=0.71$ \\
FL CV & 8.15 & 8.13 & \\
FL CV & $9.36 \pm 5.04 \mu \mathrm{m}$ & $6.84 \pm 2.21 \mu \mathrm{m}$ & $t=0.284 ; P=0.78$ \\
LT CV $_{\text {wm }}$ & 11.81 & 13.37 & \\
LT CV $_{\mathrm{bm}}$ & $8.89 \pm 4.71 \mu \mathrm{m}$ & & \\
\hline
\end{tabular}

Table 1. Descriptive statistics of sperm morphometric of the two analysed Syngnathus abaster populations. Data derived from 200 spermatozoa from Sardinian population and 180 from Venice population (20 spermatozoa per specimens); all measurements are in $\mu \mathrm{m}$ and are referred to mean \pm Standard deviation, $t$-Student and coefficient of variation (CV) calculated for all parameters within (CVwm) and between males (CVbm) of each population. Coefficient of variation between males was calculated as $\mathrm{SD} /$ mean $\times 100$ and adjusted for sample size following the formula $(\mathrm{CV} \mathrm{X}(1+(1 / 4 n)))($ Sokal and Rohlf 1995)

frequently multinucleated cells, with a unique flagellum emerging from each nucleus (Fig. 1D,E). Moreover, several abnormal sperm, such as those having a very short flagellum, were observed, but their unusual appearance (Fig. 1F) was clearly related to a damage during handling (i.e. the flagellum appeared clearly broken, because lacking of its terminal end).

The morphometric analysis of full-developed germ cells evidenced that the differences in the average value of the four sperm parameters analysed in the two populations were not statistically significant (Table 1 ). The average values of three characters (width and length of the head and length of the flagellum) measured in all males of the two populations (Figs. 2-4) show a variation range equal:

1 from 0.68 to $0.85 \mu \mathrm{m}$ for the average width of the head in the Sardinian population and $0.63-0.76 \mu \mathrm{m}$ in the Venice population (Fig. 2);

2 from 3.02 to $3.52 \mu \mathrm{m}$ for the average length of the head in the Sardinian population and 3.06-3.87 $\mu \mathrm{m}$ in the Venice population (Fig. 3);

3 from 51.04 to $60.72 \mu \mathrm{m}$ for the average length of the flagellum in the Sardinian population and 51.67-57.24 $\mu \mathrm{m}$ in the Venice population of (Fig. 4).
Moreover, the total length of the spermatozoa in both populations showed a range of sizes (minimum-maximum) of $45-80 \mu \mathrm{m}$ for the Sardinian population and $50-75 \mu \mathrm{m}$ to that of Venice, although, most of mature sperms analysed had total length between 55 and $65 \mu \mathrm{m}$ in both populations (Fig. 5).

The variability of all analysed sperm traits was high in both population, as shown by the respective coefficients of variation within and between males calculated in both population (differences were not statistically significant) (see Table 1).

Finally, as shown by the MDS analysis, sperm population within a single male, as well as those within each population and between populations, were not structured in discrete groups or classes (Figs 6 and 7).

\section{Discussion}

Data reported in the present study show that the differences in the average values of all morphometric parameters analysed between the two brackish water populations of Syngnathus abaster and tested with the $t$-Student were no statistically significant $(0.1<P \leq 0.9)$. Moreover, the stress values (the

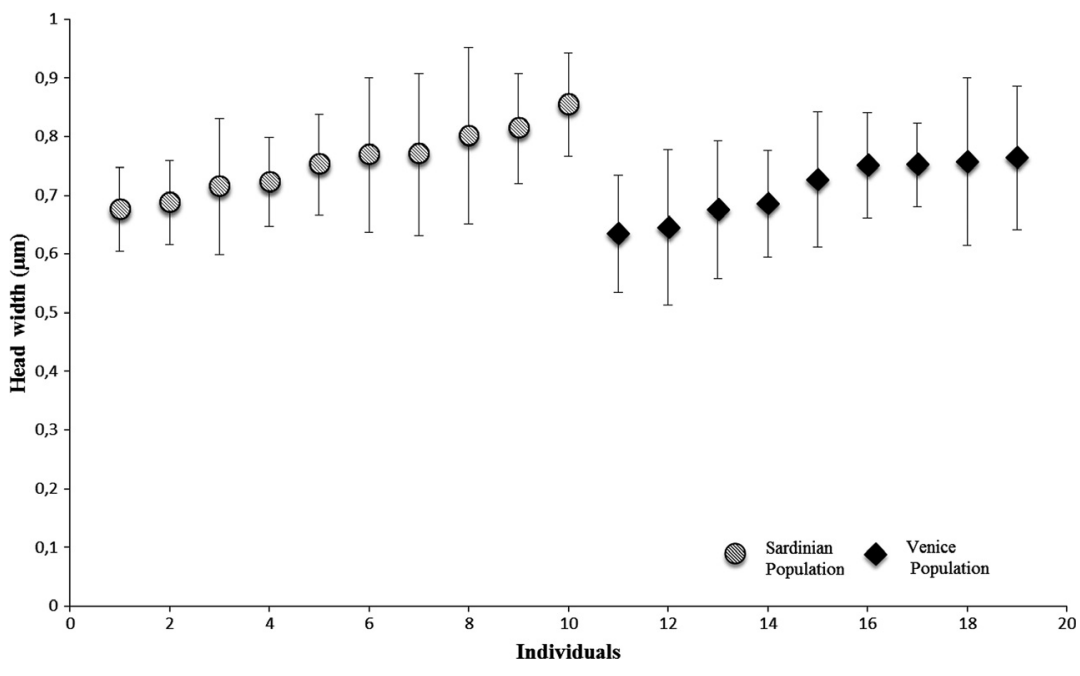

Fig. 2-Head width of Syngnathus abaster sperm calculated in each male of both Sardinian $(n=10$ specimens $)$ and Venice $(N=9)$ populations. Twenty spermatozoa were measured for each male. The mean and standard deviation are indicated by symbol (oblique strikethrough circle and black diamond) and vertical bars, respectively. Within each population, individual males are ordered by increasing mean values. 
Fig. 3- Head length of $S$. abaster sperm calculated in each male of both Sardinian ( $n=10$ specimens) and Venice $(\mathrm{N}=9$ specimens) populations. Twenty spermatozoa were measured for each male. The mean and standard deviation are indicated by symbol (oblique strikethrough circle and black diamond) and vertical bars, respectively. Within each population, individual males are ordered by increasing mean values.

Figure 4-Flagellum length of $S$. abaster sperm calculated in each male of both Sardi$\operatorname{nian}(\mathrm{n}=10$ specimens) and Venice $(N=9$ specimens) populations. Twenty spermatozoa were measured for each male. The mean and standard deviation are indicated by symbol (oblique strikethrough circle and black diamond) and vertical bars, respectively. Within each population, individual males are ordered by increasing mean values.

Figure 5-Histogram represented the frequency distribution of the sperm total length in both populations. $(N=200$, in Sardinian population and $N=180$ in Venice population).
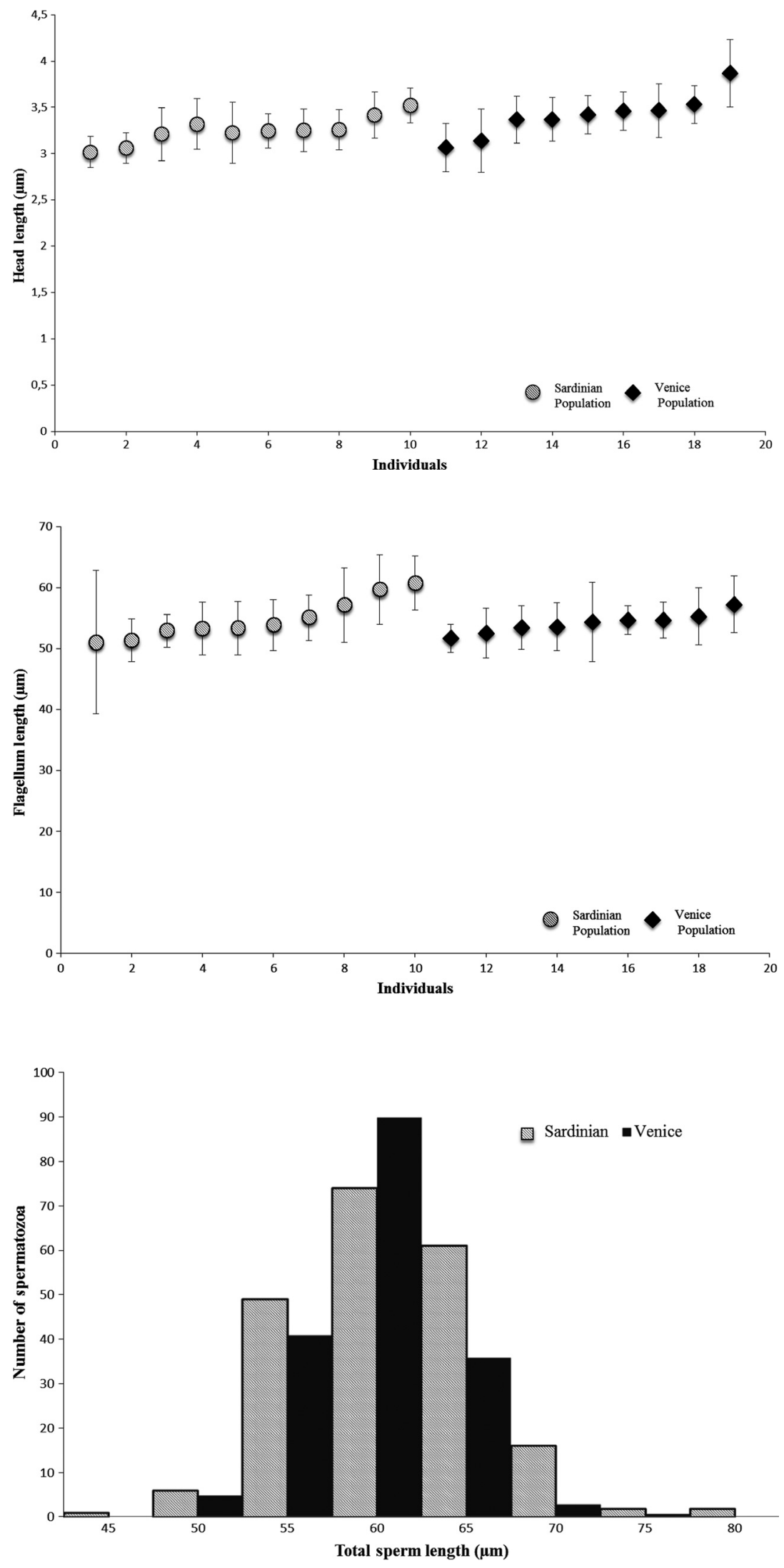

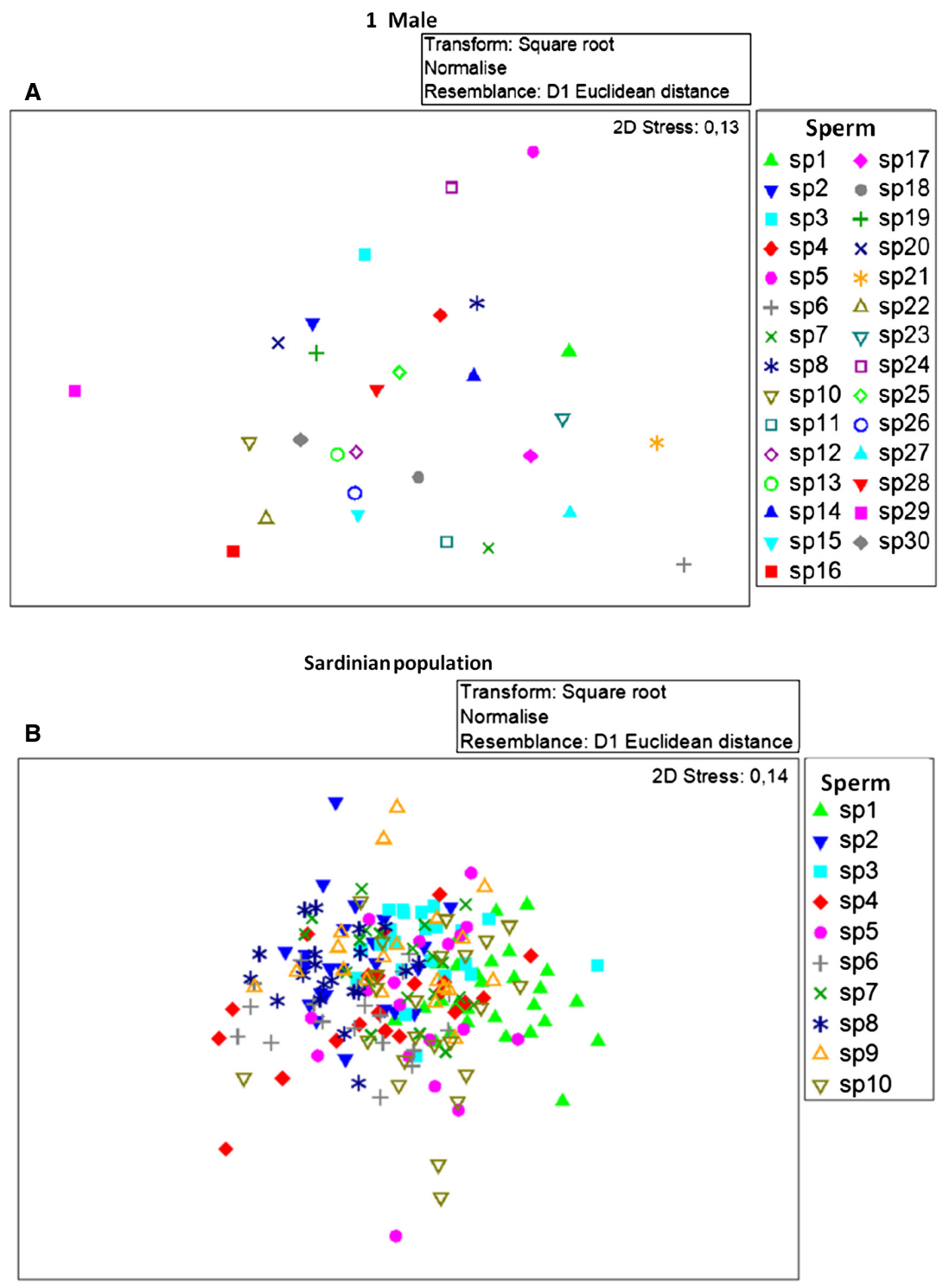

Figure 6-Multidimensional scaling plots regarding within a single Sardinian male $(N=30)($ A) and within Sardinian population sperm data $(N=200)(B)$. measure for a goodness-of-fit statistic) of all the MDS analyses here performed are $0.1<$ Stress values $\leq 0.2$. These values, which correspond to a good representation of the data without a real danger of bad interpretation, allow as to assert that (i) the two populations seem to be indistinguishable on the base on their spermatozoa (stress value $=0.16$ ) and (ii) there is no polymorphism, being sperm not distinguishable into discrete classes either within a single male (stress value $=0.13$ ) or within each populations (stress values $=0.14$ and 0.2 for Sardinian and Venice populations, respectively). The latter datum does not seem to support the presence of polymorphic sperm, classified in three different groups, reported in the freshwater population of the same species $S$. abaster (Dzyuba et al. 2008).

First, the two studies differ in the methods performed to obtain measurable sperm. We used fixed spermatozoa, whereas Dzyuba et al. (2008) used living cells. In addition, we have measured mature (apparently fully formed) and intact sperm (sperm with all their three portions clearly visible, i.e. nucleus, midpiece and flagellum) only, whereas Dzyuba et al. (2008) measured all sperm when it was possible to obtain clear images. Because these methodological differences, spermatozoa with very short flagellum were discarded in our study, whereas Dzyuba et al. (2008) included them in their data set, despite the analysis on motility of these sperm had a negative outcome. This could explain, for example, the high difference in the minimum value of total sperm length obtained in the two studies; 45 and $55 \mu \mathrm{m}$ in our study for Sardininan and Venice population, respectively, and $11 \mu \mathrm{m}$ for the Ukrainian freshwater population. However, of the three morph types showed by the Dzyuba et al. (2008), only the spermatozoa with flagella longer than $40 \mu \mathrm{m}$ were mobile 


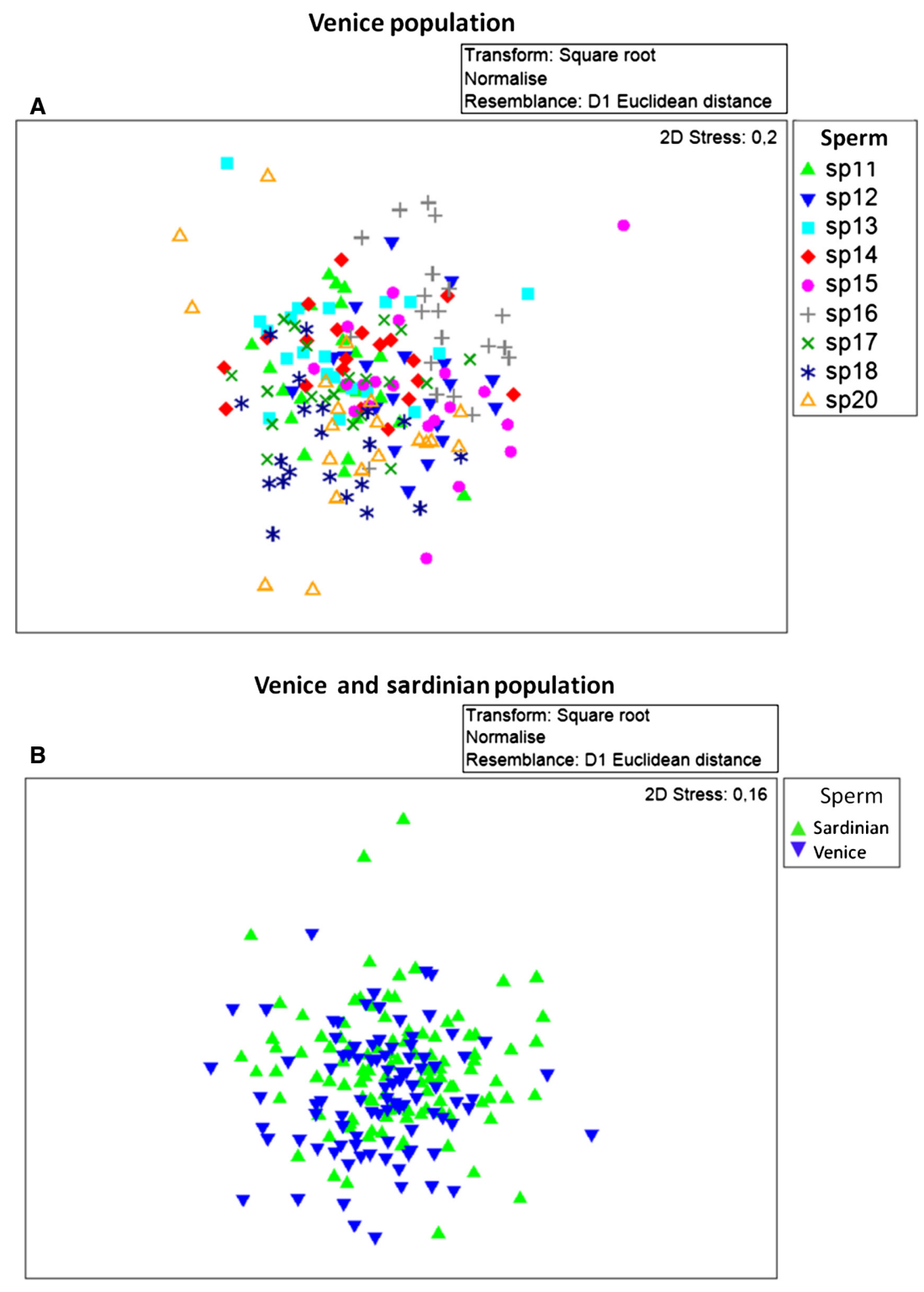

Figure 7-Multidimensional scaling plots regarding Venice population (A) $(N=180)$ and Sardinian and Venice populations sperm data $(N=200$ obtained pooling 100 randomly selected sperm from each population) (B).

and most mobile sperm were longer than $60 \mu \mathrm{m}$. The latter datum seems to support our criteria in choosing of mature and intact spermatozoa only, most of which had total length between 55 and $65 \mu \mathrm{m}$ in both populations.

Second, the two studies differ in the number of sperm measured per male. In our study, we measured 20 sperm per male for each of 10/9 males per population, whereas Dzyuba et al. (2008) measured a pool of only 93 spermatozoa obtained by 14 sperm samples. Although the authors did not specify the numbers of sperm per each males, their average was less than 10 sperm per male. Because of that, in our study, the variability in sperm traits both within male and between males of the same population has been estimated, whereas, Dzyuba et al. (2008) estimated only the variability between males.
Last, but not least, the two studies use a different form of $S$. abaster species. We have analysed the of brackish water form whereas Dzyuba et al. (2008) used the freshwater one. This species lives in both brackish water and freshwater, as well as seawater, and even if marine and freshwater forms appear morphologically identical, there is still not unequivocal genetic evidence that they are the same species.

Apart from the considerations listed above, there is more than one difficulty in considering the different types of sperm reported in syngnathids species as heteromorphism:

1 Generally, parasperm clearly differ from eusperm in both morphology and/or size. On the contrary, the various morphs reported in S. abaster by Dzyuba et al. (2008) are much overlapped, making unambiguous categorization of 
each sperm class difficult, thus assessing their respective function and potential adaptive significance problematical.

2 As mentioned in the introduction, the spermatozoa of acquasperm type, reported in the seminal fluid of Syngnathus schlegeli and Hippocampus kuda (Watanabe et al. 2000; Van Look et al. 2007) could be either parasperm or, more likely, developing spermatids. The semicystic spermatogenetic process causes a precocious release from the spermatocysts of germ cells, which complete their development within the lumen of testis (Carcupino et al. 1999;. Biagi et al. 2015). Semicystic spermatogenesis leads to a reduced number of simultaneously mature sperm, and this has been interpreted as one of the possible mechanisms evolved to reduce the cost of sperm production. This is particularly true in those species that, like syngnathids, perform male parental care. In these species, it has been speculated that the male cannot invest all the energies devoted to reproduction in sperm production (Manni and Rasotto 1997; Giacomello et al. 2008). However, no data on the occurrence of semicystic spermatogenesis in Hippocampus species are known, so that further studies on the spermatogenetic process in these syngnathids are needed to clarify the nature of parasperm reported in $H$. kuda.

3 The reason why parasperm are produced has not been fully elucidated, and their relationship to the reproductive mode is uncertain. However, the most likely function for invertebrates parasperm has been hypothesized to be correlated to competition, that is parasperm may be available for males to overcome sperm competition. Sperm competition occurs between sperm from two or more males to fertilize ovulated or spawned eggs from a single female (Parker 1970). Therefore, sperm emitted from multiple males compete each other for eggs. It is predicted that sperm competition may result in elimination or displacement of eusperm from previous males, blocking or destruction of the non-kin eusperm (soldier sperm; Kura and Nakashima 2000), and preventing or delaying further mating of females. A possible role correlated to sperm competition has been also reported by Hayakawa (2007), for the unflagellate parasperm in a non-copulatory marine cottoid. It has been experimentally demonstrated that parasperm of this teleost accumulate at the boundary surface of an egg mass where ovarian fluid contacts seawater and that parasperm lumps are obstacles to the late-arriving sneakers eusperm.

Males of all syngnathid species (with or without a brood pouch) have exclusive paternity of their young, so no sperm competition seems to be present (Avise et al. 2002; Ah-King et al. 2006). Lack of sperm competition is also supported by the very low sperm concentration (much lower than estimated for other fish species) (Van Look et al. 2007; Biagi et al. 2008; Dzyuba et al. 2008). The functional sperm: egg ratio has been estimated to be about $70: 1$ in the brackish water form of
S. abaster (Biagi et al. 2008) lower, $191: 1$ in freshwater form of the same species (S. abaster) (Dzyuba et al. 2008) and even much lower 5:1 in Hippocampus kuda (Van Look et al. 2007). These values are numerous orders of magnitude lower than estimated in the zebrafish (Danio rerio) (48 $000: 1$ ), which was considered to have one of the lower sperm concentration in fish (Stockley et al. 1996).

Due to both the low sperm concentration and high reproductive efficiency of males (most of the carried eggs are fertilized) (personal observation), it could be speculated that all sperm, produced by Syngnathidae, must be functional and free of errors. Despite this, both populations of $S$. abaster here analysed exhibit a high variation in all sperm traits, both among individual sperm within an ejaculate and among males. However, sperm variability is not only associated to heteromorphism or the spermatogenetic errors. Recently, comparative studies of both passerine birds and some teleost species have showed that the variations are also present in normal spermatozoa within a species within each male of the same species. These types of variations are negatively associated with the risk of sperm competition (Calhim et al. 2007; Thünken et al. 2007; Immler et al. 2008; Kleven et al. 2008; Lifjeld et al. 2010). Sperm design is commonly assumed to be under male and hence diploid genetic control (Beatty 1970; Parker and Begon 1993; Birkhead et al. 2005). Yet, there is increasing evidence that haploid genes of the sperm are expressed and may influence the sperm design and function (Joseph and Kirkpatrick 2004; Immler 2008). The expression of the haploid set of genes of the sperm may be one explanation for the observed variation among sibling sperm, as sibling sperm differ genetically by $50 \%$ on average. The optima for sperm design under diploid and haploid control may differ (Parker and Begon 1993); however, under increased selection pressure, for example due to intense postcopulatory sexual selection, we expect the optima under diploid and haploid scenario to be more similar. In addition, under intense selection, variation in design among sibling sperm is expected to be reduced due to directional selection. This can be interpreted as evidence of stronger stabilizing selection on males producing an optimal sperm type when the risk of sperm competition increases. Parker (1998) emphasized this principle by mentioning that the production of high quality error free spermatozoa is costly and that there will be selection against it if the costs are not equal to or outweighed by the benefits (fertilizing the oocyte). Thus, in the absence of sperm competition, there may be little benefit in investing energy on the quality of sperm production (Bauer and Breed 2006)

In conclusion, we consider that the high variability in syngnathids sperm traits does not relate to the phenomenon of polymorphism sensu stricto, but rather to the variation in design among sibling functional sperm due to a low selection pressure determined by the absence of postcopulatory sexual selection, that is absence of sperm competition. 


\section{Acknowledgements}

This work is financially supported by grant from the Regional Operational Programme (POR) of Sardinia, Europoe Social Fund (FSE) 2007-2013.

\section{References}

Ah-King, M., Elofsson, H., Kvarnemo, C., Rosenqvist, G. and Berglund, A. 2006. Why is there no sperm competition in a pipefish with externally brooding males? Insights from sperm activation and morphology. Fournal of Fish Biology 68: 1-5.

Avise, J. C., Jones, A. G., Walker, D. and DeWoody, J. A. 2002. Genetic mating systems and reproductive natural histories of fishes: lessons for ecology and evolution. Annual Review of Genetics 36: 1945.

Bauer, M. and Breed, W. G. 2006. Variation of sperm head shape and tail length in a species of Australian hydromyine rodent: the spinifex hopping mouse, Notomys alexis. Reproduction Fertility and Development 18: 797-805.

Beatty, R. A. 1970. The genetics of the mammalian gamete. Biological Reviews 45: 73-119.

Biagi, F., Addis, A., Floris, A., Corso, G., Franzoi, P., Torricelli, P. and Carcupino, M. 2008. Sperm number and structure of the black-striped pipefish Syngnathus abaster (Teleostei, Syngnathidae). In Collodel, G. and Moretti, E. (Eds): Sperm Morphology and Pathology, pp. 1-11. Research Signpost, Kerala, India.

Biagi, F., Piras, F., Farina, V., Zedda, M., Mura, E., Floris, A., Franzoi, P., Fausto, A. M., Taddei, A. R. and Carcupino, M. 2015. Testis structure, spermatogenesis and sperm morphology in pipefishes of the genus Syngnathus. Acta Zoologica 97: 90-101.

Birkhead, T. R., Pellatt, E. J., Brekke, P., Yeates, R. and CastilloJuarez, H. 2005. Genetic effects on sperm design in the zebra fish. Nature 434: 383-387.

Calhim, S., Immler, S. and Birkhead, T. R. 2007. Postcopulatory sexual selection is associated with reduced variation in sperm morphology. PLoS ONE 2: e413.

Carcupino, M., Baldacci, A., Mazzini, M. and Franzoi, P. 1997. Morphological organization of the male brood pouch epithelium of Syngnathus abaster Risso (Teleostea: Syngnathidae), before, during and after egg incubation. Tissue and Cell 29: 21-30.

Carcupino, M., Baldacci, A., Corso, G., Franzoi, P., Pala, M. and Mazzini, M. 1999. Testis structure and symplastic spermatid formation during spermatogenesis of pipefish. fournal of Fish Biology 55: 334-353.

Carcupino, M., Baldacci, A., Mazzini, M. and Franzoi, P. 2002. Functional significance of the male brood pouch in the reproductive strategies of pipefishes and seahorses: a morphological and ultrastructural comparative study on three anatomically different pouches. Fournal of Fish Biology 61: 1465-1480.

Daisuke, T., Ryou, H., Hozi, I., Yasunori, K. and Youichi, H. 2010. Annual changes in testicular development and occurrence of parasperm in the male reproductive organs of fourspine sculpin, Cottus kazika. Ichthyological Research 57: 62-70.

Dzyuba, B. B., Van Look, K. J. W., Kholodnyy, V. S., Satake, N., Cheung, S. and Holt, W. V. 2008. Variable sperm size and motility activation in the pipefish, Syngnathus abaster; adaptations to paternal care or environmental plasticity? Reproduction Fertility and Development 20: 474-482.

Fishelson, L., Delarea, Y. and Gon, O. 2006. Testis structure, spermatogenesis, spermatocytogenesis, and sperm structure in cardinal fish (Apogonidae, Perciformes). Anatomy and Embryology 211: 3146.

Forsgren, K. L. and Young, K. A. 2009. Gonadal morphology of the weedy seadragon, Phyllopteryx taeniolatus (Lacépède): characterisation of ovarian and testicular maturation. Australian fournal of Zoology 56: 441-446.

Giacomello, E., Neat, F. C. and Rasotto, M. B. 2008. Mechanisms enabling sperm economy in blenniid fishes. Behavioral Ecology and Sociobiology 62: 271-680.

Hayakawa, Y. 2007. Parasperm: morphological and functional studies on nonfertile sperm. Ichthyological Research 54: 111-130.

Healy, J. M. and Jamieson, B. G. M. 1981. An ultrastructural examination of developing and mature paraspermatozoa in Pyrazus ebeninus (Mollusca, Gastropoda, Potamididae). Zoomorphology 98: 101119.

Herald, E. S. 1959. From pipefish to seahorse - a study of phylogenetic relationships. Proceedings of the California Academy of Sciences 29: 465-473.

Immler, S. 2008. Sperm competition and sperm cooperation: the potential role of diploid and haploid expression. Reproduction 135: 275-283.

Immler, S., Calhim, S. and Birkhead, T. R. 2008. Increased postcopulatory sexual selection reduces the intramale variation in sperm design. Evolution 62: 1538-1543.

Jamieson, B. G. M. 1987. A biological classification of sperm type, with special reference to annelids and molluscs, and example of spermiocladistics. In Mohri, H. (Ed.): New Horizons in Sperm Cell Research, pp. 311-332. Gordon and Breach sci. publ., New York.

Jamieson, B. G. M. 1991. Fish Evolution and Systematics: Evidence from Spermatozoa, pp. 319. Cambridge University Press, Cambridge.

Joseph, S. B. and Kirkpatrick, M. 2004. Haploid selection in animals. Trends in Ecology \& Evolution 19: 592-597.

Kleven, O., Laskemoen, T., Fossøy, F., Robertson, R. J. and Lifjeld, J. T. 2008. Intraspecific variation in sperm length is negatively related to sperm competition in passerine birds. Evolution 62: 494499.

Kura, T. and Nakashima, Y. 2000. Conditions for the evolution of soldier sperm class. Evolution 54: 72-80.

Lahnsteiner, F. 2003. The spermatozoa of the cardinal fish. Fournal of Fish Biology 62: 115-128.

Laskemoen, T., Kleven, O., Fossøy, F. and Lifjeld, J. T. 2007. Intraspecific variation in sperm length in two passerine species, the bluethroat Luscinia svecica and the willow warbler. Phylloscopus trochilus. Ornis Fennica 84: 131-139.

Lifjeld, J. T., Laskemoen, T., Kleven, O., Albrecht, T. and Robertson, R. J. 2010. Sperm length variation as a predictor of extrapair paternity in passerine birds. PLOS ONE 5: e13456.

Manni, L. and Rasotto, M. B. 1997. Ultrastructure and histochemistry of the testicular efferent duct system and spermiogenesis in Opistognathus whitehurstii (Teleostei, Trachinoidei). Zoomorphology 117: 93-102.

Mattei, X. 1993. Peculiarities in the organization of testis of Ophidion sp. (Pisces: Teleostei). Evidence for two types of spermatogenesis in teleost fish. Fournal of Fish Biology 43: 931-937.

Mattei, C. and Mattei, X. 1984. Spermatozoides biflagelles chez un poisson teleosteen de la famille des Apogonidae. Fournal of Ultrastructure Research 88: 223-228.

Monteiro, N. M., Almada, V. C. and Vieira, M. N. 2005. Implications of different brood pouch structures in syngnathid reproduction. Fournal of the Marine Biological Association of the United Kingdom 85: 1235-1241. 
Parker, G. A. 1970. Sperm competition and its evolutionary consequences in the insects. Biological Revierws 45: 525-567.

Parker, G. A. 1998. Sperm competition and the evolution of ejaculates: towards a theory base. In Birkhead, T. R. and Møller, A. P. (Ed.): Sperm Competition and Sexual Selection, pp. 3-54. Academic Press, London.

Parker, G. A. and Begon, M. E. 1993. Sperm competition games: sperm size and number under gametic control. Proceedings of the Royal Society of London Series B 253: 255-262.

Sokal, R. R. and Rohlf, F. J. 1995. Biometry: The Principles and Practice of Statistics in Biological Research, 3rd Edn. pp. 887. W.H. Freeman and CO., New York.

Stockley, P., Gage, M. J. G., Parker, G. A. and Møller, A. P. 1996. Female reproductive biology and the coevolution of ejaculate characteristics in fish. Proceedings of the Royal Society of London Series B-Biological Sciences 263: 451-458.

Swallow, J. G. and Wilkinson, G. S. 2002. The long and short sperm polymorphism in insects. Biological Reviews 77: 153-182.

Tachi, C. 1990. Introduction to Reproductive Biology. University of Tokyo Press, Tokyo.

Thünken, T., Bakker, T. C. M. and Kullmann, H. 2007. Extraordinarily long sperm in the socially monogamous cichlid fish Pelvicachromis taeniatus. Naturwissenschaften 94: 489491.

Till-Bottraud, I., Joly, D., Lachaise, D. and Snook, R. R. 2005. Pollen and sperm heteromorphism: convergence across kingdoms? fournal of Evolutionary Biology 18: 1-18.

Van der Horst, G., Maree, L., Kotze, S. H. and O'Riain, M. J. 2011. Sperm structure and motility in the eusocial naked mole-rat Heterocephalus glaber: a case of degenerative orthogenesis in the absence of sperm competition? BMC Evolutionary Biology 11: 351.

Van Look, K. J. W., Dzyuba, B., Cliffe, A., Koldewey, H. J. and Holt, W. V. 2007. Dimorphic sperm and the unlikely route to fertilisation in the yellow seahorse. Fournal of Experimental Biology 210: 432-437.

Watanabe, S., Kaneko, T. and Watanabe, Y. 1999. Immunocytochemical detection of mitochondria-rich cells in the brood pouch epithelium of the pipefish, Syngnathus schlegeli: structural comparison with mitochondria-rich cells in the gills and larval epidermis. Cell and Tissue Research 295: 141-149.

Watanabe, S., Hara, M. and Watanabe, Y. 2000. Male Internal Fertilization and Introsperm-like Sperm of the Seaweed Pipefish (Syngnathus schlegeh). Zoological Science 17: 759-767. 